\title{
Complex Innovative Design for NASH Clinical Trials
}

\author{
Shein-Chung Chow* \\ Department of Biostatistics and Bioinformatics, Duke University School of Medicine, USA
}

*Corresponding author: Shein Chung Chow, Department of Biostatistics and Bioinformatics, Duke University School of Medicine, Durham, North Carolina, USA.
Received Date: August 31, 2020

Published Date: September 14, 2020

\begin{abstract}
The prevalence of non-alcoholic fatty liver disease (NAFLD) and non-alcoholic steatohepatitis (NASH) is increasing world-wide. The development of drug product for treating NASH is considered unmet medical need. Due to the increasing prevalence and the health burden, there is a high need to develop therapeutic strategies for patients with NASH. The primary objective of the treatment for NASH is to prevent liver-related morbidity and mortality, which generally takes a long time (e.g., more than 10-20 years) to develop. In practice, it is not feasible to conduct such clinical studies. Thus, regulatory agencies such as United States Food and Drug Administration (FDA) encourage to look at surrogate endpoints for review and approval. Seamless adaptive clinical trial designs in NASH provide an opportunity to shorten the overall path to registration for a new drug and offer continuity for patients enrolled in the study. In this article, Characteristics for applying various seamless adaptive designs to clinical trials in NASH are reviewed. Statistical, clinical, and strategic issues related to drug development of NASH are also discussed.
\end{abstract}

Keywords: Endpoint selection; Population shift, Therapeutic index; Seamless adaptive design

\section{Introduction}

Non-alcoholic steatohepatitis (NASH) is defined histologically by the presence of hepatic steatosis with evidence for hepatocyte damage with or without fibrosis. The most important histological feature associated with mortality in NASH is presence of significant fibrosis. Although recent data suggest that some patients with fatty liver can progress to NASH and clinically significant fibrosis, most of the fibrosis progression seems to occur in patients with NASH. NASH has been recognized as one of the leading causes of cirrhosis in adults and NASH related cirrhosis is currently the second indication for liver transplants in the United States [1]. Non-alcoholic fatty liver disease (NAFLD) is the presence of hepatic accumulation of triglycerides in the hepatocytes in the absence of significant alcohol intake, viral infection, or any other specific etiology of liver disease. It represents a histopathologic spectrum ranging from steatosis alone to NASH, fibrosis, and cirrhosis [2,3].
The diagnosis of NASH requires a liver biopsy with subsequent confirmation of a specific histopathologic pattern. The minimal criteria for the diagnosis of steatohepatitis include the presence of $>5$ $\%$ macro vesicular steatosis, inflammation, and liver cell ballooning, typically with a predominantly centrilobular distribution in adults. The NAFLD activity score (NAS) is a validated scoring system that can be used to assess histologic change in studies of both adults and children with NASH [4]. It is an un-weighted composite of steatosis, inflammation, and ballooning scores. It should be noted that a score of 5 or more is associated with a greater likelihood of having NASH, an NAS $\geq 5$ does not necessarily confirm a diagnosis of NASH. Additionally, NAS has not been validated as a marker for likelihood of disease progression (e.g., cirrhosis, mortality) and/or response to therapy [5]. The use of NAS is currently limited to clinical trial settings. Thus, it is recommended that a validated method for the 
staging of NASH be used for assessment of changes in disease stage in clinical trials of NASH. In practice, the NASH Clinical Research Network (CRN) fibrosis staging system is the most validated system currently available. Total score ranges from 0 to 4 (no fibrosis to cirrhosis).

Given the high unmet medical need, no universally accepted clinical endpoints, the lack of validated surrogate endpoints, and no approved drug therapy for NASH, the United States Food and Drug Administration (FDA) published guidance to assist the sponsor in drug development of NASH [6]. FDA's guidance provided some general considerations including (i) specify criteria that establish a diagnosis of cirrhosis, e.g., a diagnosis of cirrhosis should be supported by histology such as a NASH Clinical Research Network (CRN) fibrosis score of 4, (ii) stratified randomization, e.g., patients with type 2 diabetes mellitus or patients with NASH-cirrhosis who are treated with Vitamin E or pioglitazone, (iii) sufficient duration and adequate sample size, and (iv) establishment of expert committee to adjudicate cases for safety. In addition, FDA encourages the use of biochemical or imaging non-invasive biomarkers that can replace liver biopsies.

Along this line, it is suggested that a seamless adaptive design be used in NASH clinical studies [7,8]. A seamless adaptive design provides the flexibility and efficiency for identifying potential signals of clinical benefit of the test treatment under investigation and make prompt pre-planned adaptations without undermining the validity or integrity of the trial $[9,10]$. In NASH clinical trials, however, some practical issues inevitably occur. These issues include, but are not limited to, (i) only limited number of patients willing to have multiple liver biopsies, (ii) lack of validated surrogate endpoints, (iii) long-term exposure to assess an impact in outcomes [11].indicated the use of seamless adaptive design appear reasonable for addressing these practical issues. A continuous seamless adaptive design may reduce the overall sample size while allowing patients to continue after each one of the phases (if a multiple-stage seamless adaptive design is used).

In the next section, some general considerations in NASH drug development from regulatory perspectives are described. Section 3 outlines some limitations and challenging issues that are commonly encountered in the conduct of NASH clinical trials. Section 4 discusses the application of seamless adaptive design in NASH clinical studies. Some concluding remarks are given in the last section of this article

\section{Regulatory Perspectives}

As indicated earlier, there is not only unmet medical need for treatment of NASH, butalso there is no approved drugs for treatment of NASH in the United States due to complexity of the disease. Thus, to ensure the success of clinical development for NASH, some strategic frameworks are necessary implemented. These strategic frameworks include potential surrogate endpoints as well as statistical and logistical approaches that could be considered for applying seamless adaptive designs to clinical trials in NASH with the goal of facilitating drug development for this growing unmet medical need. In this section, general considerations for development of treatment for NASH are discussed from regulatory perspectives.

\section{Regulatory guidance}

In December, 2018, FDA issued draft guidance on developing drugs to treat patients who have NASH with liver fibrosis to assist the sponsor in developing NASH drug products. In practice, given the high prevalence of NASH, the associated morbidity, the growing burden of end-stage liver disease, and the limited availability of livers for organ transplantation, the FDA draft guidance provided recommendations for pre-clinical and clinical development including trial design and endpoint selection to support regulatory approval of drugs to treat non-cirrhotic NASH. The FDA draft guidance meant to assist the sponsor in identifying therapies that will slow the progress, halt, or reverse NASH and NAFLD will address an unmet medical need. However, FDA indicated that the guidance is not meant to cover the development of drugs to treat cirrhosis caused by NASH or the development of in vitro diagnostics that may be used in developing drugs to treat the disease.

\section{General considerations}

As stated in the draft guidance, NAFLD consists of three successive stages: non-alcoholic fatty liver (NAFL), noncirrhotic $\mathrm{NASH}$, and NASH with cirrhosis. The draft guidance provides sponsors a convenient conceptual framework to identify areas of potential future drug development. However, because patients' NAFL can exist for many years and may not progress to NASH, it may be challenging to demonstrate a favorable benefit-risk profile of pharmacological treatment(s) in NAFL patients. Thus, it is suggested that sponsors should consider the following general considerations during drug development for treatment of noncirrhotic NASH with liver fibrosis [6]. First, the sponsor should consider using animal models for NASH to screen and identify potential investigational drugs. The sponsor should select a specific animal model based on the mechanism of action of the investigational drug. Second, if there is a potential for liver toxicity based on animal toxicology studies, the sponsor should institute an appropriate plan to monitor liver safety early in drug development. For such a plan, the sponsor should consider the challenges of effectively recognizing a liver signal in a chronic liver condition such as NASH.

As stated in the guidance, until a sponsor can characterize a drug's initial tolerability, preliminary safety, and pharmacokinetics, patients with evidence of abnormal liver synthetic function should 
be excluded from early phase trials (i.e., phase 1 and early proof-ofconcept (POC) clinical trials). In addition, the sponsor should study the effects of hepatic impairment on the drug's pharmacokinetics early during the drug development program in a dedicated hepatic study to support appropriate dosing and dose adjustment across the spectrum of NASH liver disease.

\section{Specific considerations}

In addition to general considerations, the guidance also provided specific considerations regarding phase 2 development considerations, phase 3 development considerations and pediatric considerations, which are summarized below. For phase 2 and phase 3 studies, FDA suggested that sponsors should enroll patients with a histological diagnosis of NASH with liver fibrosis made within six months of enrolment, taking into consideration patients' standard of care and background therapy for other chronic conditions. FDA also says that patients' weight should be stable for three months prior to enrolment. FDA also indicated that phase 3 studies for NASH should be double-blind and placebo-controlled with the goal of slowing, halting, or reversing disease progression and improving clinical outcomes.

Because of the slow progression of NASH and the time required to conduct a trial that would evaluate clinical endpoints such as progression to cirrhosis or survival, the FDA recommends sponsors consider several liver histological improvements as endpoints reasonably likely to predict clinical benefit to support accelerated approval under the regulations. FDA recommended endpoints include (i) resolution of steatohepatitis on overall histopathological reading and no worsening of liver fibrosis on NASH CRN fibrosis score, (ii) improvement in liver fibrosis greater than or equal to one stage (NASH CRN fibrosis score) and no worsening of steatohepatitis (defined as no increase in NAS for ballooning, inflammation, or steatosis), or (iii) both resolution of steatohepatitis and improvement in fibrosis. The FDA guidance also provided some caveats for pediatric development. As indicated in the FDA guidance, pediatric NASH appears to have different histological characteristics as well as a different natural history when compared to adult NASH. For reasons that are currently unknown, disease characteristics and progression in pediatric patients may be different.

\section{Accelerated/conditional marketing approvals}

In practice, FDA has adopted policies to expedite drug development for serious medical conditions where few or no therapies exist. This is an evolving process which began in the 1980's with drugs intended to treat HIV, and continues through the present with multiple regulatory initiatives to facilitate availability of new drugs to patients - these programs either tackle the issue of accelerating clinical development and/or accelerating reviews by the regulatory agencies. For example, in 2014, FDA issued a guidance describing "Expedited Programs for Serious Conditions" which consolidated information on Fast Track, Breakthrough Therapy, Accelerated Approval, and Priority Review [12]. Similarly, in 2014, the EMA launched Adaptive Licensing which, like the US Accelerated Approval Pathway, is also aimed at accelerating marketing approvals for products throughout the European Union [13]. For development of products to treat NASH these accelerated pathways offer the potential to use surrogate endpoints to obtain Accelerated (US) and conditional (EU) marketing approval, with full marketing approval being granted with subsequent confirmatory studies using well established, and well-defined clinical outcomes.

With a registration pathway involving a two-stage process namely, conditional marketing approval using surrogate endpoints, followed by full marketing approval using well-defined clinical outcomes - drug development programs to treat NASH seem well suited to continuous adaptive clinical studies. Conditional/ accelerated marketing authorization are not new concepts. For example, in the 1980s accelerated marketing approvals were granted for a number of drugs to treat HIV based on surrogate endpoints, with post approval studies being required for full approval. Indeed, in 1997 Sheiner's disruptively innovative paper describing a learn-confirm strategy suggested we move away from thinking in terms of separate Phase $1 / 2 / 3$ studies [14]. Although Sheiner's paper focused on early studies, the concept has been applied across the development continuum - namely, initial studies to learn about the drug and later studies to confirm a positive benefit-risk profile. There are, however, a number of stakeholders to be considered when designing seamless adaptive studies (patients, regulators, payers), and clearly one size does not fit all medical situations. However, given the challenges in developing therapies for NASH, it seems reasonable to assume that seamless adaptive designs provide a good fit in terms of development for marketing authorizations, and that these adaptive designs will continue to evolve as we learn more about this emerging epidemic.

\section{Limitations and Challenges in NASH Clinical Trials}

Due to the complexity of the diseases, there are several limitations in the conduct of NASH clinical trials. These limitations include the lack of accurate, reproducible, and easily applied methods to assess NASH which creates major challenges not only for drug development, but also in the clinical management of NASH patients.

\section{Disease assessment}

Liver biopsy remains the gold standard for the diagnosis of NASH but it has several limitations. There is always a risk that the biopsy taken might not be representative for the amount of fibrosis in the whole liver. Increasing the length of liver biopsy decreases 
the risk of sampling error. In general, except for cirrhosis, for which micro-fragments may be sufficient, a $25 \mathrm{~mm}$ long biopsy is considered an optimal specimen for accurate evaluation, though 15 $\mathrm{mm}$ is considered sufficient. Not only the length but also the caliber of the biopsy needle is important in order to obtain a piece of liver of adequate size for histological evaluation: a 16-gauge needle is considered appropriate for percutaneous liver biopsy [3]. Interobserver variation is another limitation which is related to the discordance between pathologists in biopsy interpretation. This can be as high as $25 \%$ but the variation is less pronounced when the biopsy assessment is done by specialized liver pathologists.

Beside the technical problems with biopsy, the procedure is unpleasant for the patient, costly, and carries a risk of rare but potentially life-threatening complications-this limits the use of liver biopsy for mass screening. Due to these limitations, the fact that NASH is usually asymptomatic and that patients usually have normal liver aminotransferases, most NASH patient are undiagnosed. It has been reported that only a minority (less than $25 \%$ ) of academic gastroenterologists and hepatologists in the USA routinely perform liver biopsies in patients with presumed NASH [15]. This level of undiagnosed patients and the need of two liver biopsies within a 48-72 week period establish a significant hurdle in the enrolment of patients in clinical trials.

\section{Study objectives and endpoints}

The primary objective of the treatment for NASH is to prevent liver-related morbidity and mortality, due mainly to the development of cirrhosis, which generally takes more than 10-20 years to develop. Due to this long natural history, there is a need of surrogate markers of avoidance of cirrhosis and thus liver-related mortality. The main predictor of disease progression is increasing fibrosis. On the other hand, patients with steatohepatitis are more likely to have a progressive disease than patients with isolated fatty liver. Therefore, complete resolution of NASH (i.e. absence of ballooning with no or minimal inflammation) with no worsening of fibrosis, or actual improvement in fibrosis are recommended as "surrogate endpoints, reasonably likely to predict clinical benefit in progression to cirrhosis and liver-related death" (Table 1). Ideally, a co-primary endpoint of two composite endpoints, 1-complete resolution of NASH with no worsening of fibrosis and, 2-at least one-point improvement in the fibrosis stage with no worsening of steatohepatitis, should be demonstrated in the marketing authorization trial. However, the clinical outcome study, aiming to demonstrate a reduction in progression to cirrhosis and portal hypertension/cirrhosis related events needs to be demonstrated.

For dose ranging trials, the histology endpoint of improvement in activity as assessed by a reduction in at least 2 points in NAS (including at least 1 point in ballooning or inflammation) is an acceptable surrogate marker of improvement. It is important to note that though NAS has proven useful for comparative analyses and interventional studies it does not provide information about fibrosis or the location of lesions. Therefore, the reduction in NAS must be associated with lack of progression in fibrosis. Biopsy driven endpoints are, in general, not feasible in a 12-24-week POC trials. In short-term POC studies, designed mainly to assess tolerability of new drugs and to look for futility signals to direct decisions regarding further development, an improvement of hepatic steatosis, as determined by magnetic resonance technology, might be suitable since improvement in steatohepatitis is generally associated with a reduction in liver fat [3]. Improvement in liver aminotransferase and other non-invasive biomarkers of insulin sensitivity, inflammation, apoptosis, and fibrosis could be helpful to evaluate the efficacy of the compound and support decision making. However, it is important to note that the use of non-invasive biomarkers methods is still considered experimental and there are no validated non-invasive biomarkers.

\section{Target population}

Prevention of cirrhosis and demonstrating a positive effect on well-defined liver outcomes are key clinical goals when considering a NASH drug development program. Therefore, for trials aiming to support marketing application, it is important that subjects with the greatest risk of progression to cirrhosis are enrolled (Table 1). Among individual features, liver fibrosis has proven the best independent association with liver-related mortality. Patients with NASH develop progressive fibrosis in $25 \%$ to $50 \%$ of individuals over $4-6$ years, while $15 \%$ to $25 \%$ of individuals with NASH can progress to cirrhosis [16]. In another study, with a mean follow-up of 13 years, $13.3 \%$ of NASH patients with mild to moderate fibrosis (stage 1-2) and $50 \%$ of patients with fibrosis stage 3 at inclusion developed cirrhosis [17]. Since in patients with NASH and advanced fibrosis (F2-F3) the probability of developing cirrhosis is much higher than in patients with early fibrosis (F1), this population is recommended for long-term outcome trials in order to enhance the chances of demonstrating a benefit within a reasonable timeframe. The enrolment of patients with moderate /advanced fibrosis for the evaluation of long-term outcomes including progression to cirrhosis should ensure that an expected number of events, calculated based on progression rate for each fibrosis stage, are obtained based on the literature $[17-19,6,20]$ in patients with NASH and advanced fibrosis (F2-F3) this progression rate can be estimated at $8 \%$ per year for fibrosis stage 3 , and $6 \%$ per year for fibrosis stage 2. Since the progression rate in some patients with mild fibrosis with additional risk factors of progression (e.g. presence of type 2 diabetes, the metabolic syndrome, high transaminases) might be fast, it is worth exploring this subgroup of patients, as an additional exploratory group.

A broad population of NASH patients including those ones with mild fibrosis is acceptable in dose ranging (phase 2) trials. However, it is recommended that a sufficient number of patients with 
moderate and severe fibrosis are enrolled in order to get preliminary data to inform the trial/s to support marketing application. Ideally, in early proof of concept trials, the target population should also be patients with biopsy confirmed NASH. However, patients at high risk of NASH, namely patients with fatty liver and diabetes and /or the metabolic syndrome with or without high liver enzymes can be acceptable at this stage. Non-invasive serum biomarkers o imaging can be used to enrich a population in a POC trial.

\section{Adaptive Design Approach in NASH Clinical Trials}

For development of drug products treating patients with NASH, the following trials are necessarily conducted (i) early phase trials/ proof-of-concept, (ii) phase 2 dose ranging, (iii) phase 3 trials, and (iv) phase 4 post-marketing study. To speed up and shorten the development process, the sponsor may be interested in considering the following seamless adaptive design for the development of the drug product for NASH: (i) proof-of-concept/dose ranging adaptive trial design, (ii) phase $3 / 4$ adaptive trial design, and (iii) phase 2/3/4 adaptive design. In addition, to ensure the success of NASH clinical studies utilizing seamless adaptive trial design, it is suggested that an investigator's wish list should be provided.

\section{Investigator's wish list}

For NASH clinical trials, the following is a typical wish list from PI or sponsor's perspectives.

1. Be able to make decision early.

2. Use biomarkers or surrogate endpoints rather than regular clinical endpoints.

3. Option for sample size re-estimation.

4. Be able to drop inferior arms.

5. Be able to add additional arms.

6. Response-adaptive randomization after interim analyses.

To account for investigator's wish list, it is suggested a multiplestage seamless adaptive design with potential adaptations after the review of accumulated data at interim be considered.

\section{Two-stage seamless adaptive trial design}

One of the most commonly considered adaptive designs is probably a multiple-stage seamless adaptive design that combines several independent studies into a single study that can addresses study objectives of the intended individual studies. For two-stage adaptive designs, four categories have been reported depending upon their study objectives and study endpoints at different stages. These categories include (i) design with same study objectives and study endpoints at different stages, (ii) designs with same study objectives but different study endpoints at different stages, (iii) designs with different study objectives but same study endpoints at different stages, and (iv) designs with different study objectives and different study endpoints at different stages (Table 2). As it can be seen from (Table 2), depending upon whether the study objectives and the study endpoints at different stages are the same or not, there are four different types of two-stage seamless adaptive designs, namely SS, SD, DS, and DD designs for NASH clinical trials.

\section{Analysis of two-stage seamless adaptive designs}

In practice, SS design is referred to as "0-D design", while SD and DS designs are considered "1-D design". The DD design is referred to as a "2-D design". In this article, we will refer to these designs as a "k-D design", where $\mathrm{k}=0,1$, or 2 . The greater $\mathrm{k}$ is, the more complicated and problematic the seamless adaptive design is. Statistical analyses for the "k-D design", where k=0,1, or 2 are brief described below.

\section{The “0-D Design” (SS Design)}

SS Design is a two-stage seamless adaptive design with the same study objective and same study endpoint at different stages, which is similar to typical group sequential design with a planned interim analysis. Thus, standard statistical methods such as MIP (method of individual p-values), MSP (method of sum of p-values), and MPP (method of product of p-values) for group sequential design can be directly applied [21]. It should be noted that if additional adaptations such as change in primary study endpoint or hypotheses after the review of interim data, the standard methods have to be modified for the control of the overall type I error rate.

\section{The “1-D Design" (SD Design or DS Design)}

Since a "1-D design" could be an SD design or a DS design. Statistical analyses for an SD design and a DS design are different. To have a valid statistical analysis, some assumptions are necessarily made. For example, for an SD design (i.e., study objectives at different stages are the same but the study endpoints are different at different stages), it is assumed that study endpoint (e.g., a biomarker, a surrogate endpoint, or a clinical endpoint with a short duration) at the first stage is predictive of the study endpoint (i.e., regular clinical endpoint) at the second stage [22]. On the other hand, for a DS design (i.e., study objectives at different stages are different but the study endpoints at different stages are the same), we have to consider testing two sets of hypotheses at different stages.

\section{The “2-D Design” (DD Design)}

For the DD design (i.e., both study objectives and study endpoints at different stages are different), the following primary assumption and consideration are necessarily made for obtaining a valid statistical test using different endpoints for achieving study objectives at different stages: (i) study endpoint at the first stage is predictive of the study endpoint at the second stage, and (ii) consider testing two sets of hypotheses at different stages. 
Illustrated statistical analysis for a DD design using an example concerning a clinical trial for evaluation of safety, tolerability, and efficacy of a test treatment for patients with hepatitis $\mathrm{C}$ virus (HCV) infection. In the HCV study, a two-stage seamless adaptive design is considered. The trial design was to combine two independent studies (one phase $2 \mathrm{~b}$ study for treatment selection and one phase 3 study for efficacy confirmation) into a single study. Thus, study objectives at different stages are similar but different. For the study endpoint, the well-established clinical endpoint is the sustained virologic response (SVR) at week 72 (i.e., 48 weeks of treatment plus 24 weeks of follow-up). Since the PI or sponsor is interested in making early decision for treatment selection at Stage 1 . The clinical endpoint of early virologic response (EVR) at week 12 is considered as a surrogate endpoint for treatment selection at Stage 1 . Thus, the study endpoints at different stages are different. Statistical test was ten derived based on the primary assumption and consideration for addressing the study objectives at different stages.

\section{Remarks}

In the previous sections, we only consider 4 types of two-stage seamless adaptive designs based on whether study objectives and study endpoints at different stages are the same or not. In NASH clinical trials, however, it is much more complicated. As it can be seen from (Table 1), in addition to study objectives and study endpoints, the target patient populations at different stages could be different. In this case, we can further classify two-stage seamless adaptive designs into eight categories depending upon whether the target patient population is the same or not. In other words, (Table 2) can be expanded to (Table 3).

Table 1: Objectives, Endpoints and Populations in NASH Clinical Trials.

\begin{tabular}{|c|c|c|}
\hline Phase (Objective) & Primary Endpoint & Target Population \\
\hline & $\begin{array}{c}\text { Composite endpoint: complete resolution of steatohepatitis and no } \\
\text { worsening of fibrosis - } \\
\text { Composite endpoint: At least one-point improvement in fibrosis with } \\
\text { no worsening of steatohepatitis (no increase in steatosis, ballooning, or } \\
\text { inflammation) }\end{array}$ & $\begin{array}{l}\text { Biopsy confirmed NASH patients with moder- } \\
\text { ate / advanced fibrosis (F2/F3) }\end{array}$ \\
\hline $\begin{array}{l}\text { Trials to support a market- } \\
\text { ing application }\end{array}$ & $\begin{array}{l}\text { Clinical outcome underway by the time of submission: } \\
\text { Histopathologic progression to cirrhosis } \\
\text { MELD score change by }>2 \text { points or MELD increase to }>15 \text { in population } \\
\text { enrolled with } \leq 13 \\
\cdot \text { Death } \\
\cdot \text { Transplant } \\
\text {-Decompensation events } \\
\text { - Hepatic encephalopathy - West Haven } \geq \text { grade } 2 \\
\text {-Variceal bleeding - requiring hospitalization } \\
\text {-Ascites - requiring intervention } \\
\text {-Spontaneous bacteria peritonitis }\end{array}$ & \\
\hline Dose ranging/Phase 2 & $\begin{array}{l}\text { Improvement in activity (NAS) / ballooning/inflammation without wors- } \\
\text { ening of fibrosis can be acceptable } \\
\text { Include a subpopulation with moderate / advanced fibrosis (F2/F3) to } \\
\text { inform PhIII }\end{array}$ & $\begin{array}{c}\text { Biopsy proven NASH (NAS } \geq 4 \text { ) } \\
\text {-Include patients with NASH and liver fibrosis } \\
\text { with any stage of fibrosis } \\
\text { Include patients with NASH and } \geq \text { Fibrosis } \\
\text { stage } 2 \text { to inform PhIII }\end{array}$ \\
\hline $\begin{array}{l}\text { Early phase trials/Proof of } \\
\text { concept }\end{array}$ & $\begin{array}{l}\text { Endpoints should be based on mechanism of drug } \\
\text { Consider using improvement in NAS (ballooning \& inflammation) and/ } \\
\quad \text { or fibrosis } \\
\text { Reduction in liver fat with a sustained improvement in transaminases }\end{array}$ & $\begin{array}{l}\text { Ideal to use patients with biopsy proven } \\
\text { NASH, but acceptable to use patients at high } \\
\text { risk for NASH (fatty liver + type } 2 \text { diabetes, the } \\
\text { metabolic syndrome and high transaminases } \\
\text { are acceptable }\end{array}$ \\
\hline
\end{tabular}

Table 2: Types of Two-Stage Seamless Adaptive Designs (Depending upon Objective and Endpoint).

\begin{tabular}{|c|c|c|}
\hline & \multicolumn{2}{|c|}{ Study Endpoint } \\
\hline Study Objectives & Same (S) & Different (D) \\
\hline Same (S) & SS & SD \\
\hline Different (D) & DS & DD \\
\hline
\end{tabular}

Table 3: Types of Two-Stage Seamless Adaptive Designs (Depending upon Objective, Endpoint, and Population).

\begin{tabular}{|c|c|c|c|c|}
\hline \multirow{2}{*}{} & \multicolumn{3}{|c|}{ Target Patient Population } \\
\cline { 2 - 6 } & & Same (S) & \multicolumn{2}{c|}{ Different (D) } \\
\cline { 2 - 6 } & & Study Endpoint & Same (S) & Different (D) \\
\hline Study Objective & Same (S) & Different (D) & SSD & SDD \\
\hline Same (S) & SSS & SDS & DSD & DDD \\
\hline Different (D) & DSS & DDS &
\end{tabular}


(Table 3) indicates that there are one "0-D design", three "1-D designs", three "2-D designs", and one "3-D design" if we account for possible shift in patient population. If there is no shift in patient population from stage to stage, (Table 3) reduces to (Table 2). In this case, statistical methods as described in the previous section can be applied. In case, there is population shift from stage to stage, in addition to the primary assumption and consideration that are necessarily made for derivation of valid statistical test under specific "1-D design" or "2-D design", an evaluation of potential shift in patient population from stage to stage need to be considered. For this purpose, we may consider the method proposed by for assessment of possible population shift from stage to stage by evaluating the sensitivity index under different models that (i) shift in location parameter is random, (ii) shift in scale parameter is random, and (iii) shifts in both location and scale parameters are random. In summary, when there is a difference in study objective, endpoint, and/or population, testing two sets of hypotheses, the assumption that the study endpoint at the first stage is predictive of the study endpoint at the second stage, and the assessment of possible population shift need to be taken into consideration for obtaining a valid statistical test for address the scientific/medical questions.

\section{An Example - Clinical Development NASH Program}

For development of drug products for treating patients with NASH, after having consulted with regulatory agency, it is suggested the following clinical trials utilizing seamless adaptive designs may be useful to shorten and speed up the process of NASH drug product development: (i) proof-of-concept/dose ranging adaptive trial design, (ii) phase $3 / 4$ adaptive trial design, and (iii) phase $2 / 3 / 4$ adaptive design. For illustration purpose, consider a single seamless phase 2/3/4 adaptive trial design allows adaptations, continuous exposure, and long-term follow-up (Figure 1). Endpoints at interim analysis are (i) reduction of at least 2 points in NAS, (ii) resolution of NASH by histology without worsening of fibrosis, and/or (iii) improvement in fibrosis without worsening of NASH. One (the most promising dose) or two doses may continue to the next phase. A post-marketing phase 4 with demonstration of improvement in clinical outcomes will lead to final marketing authorization.

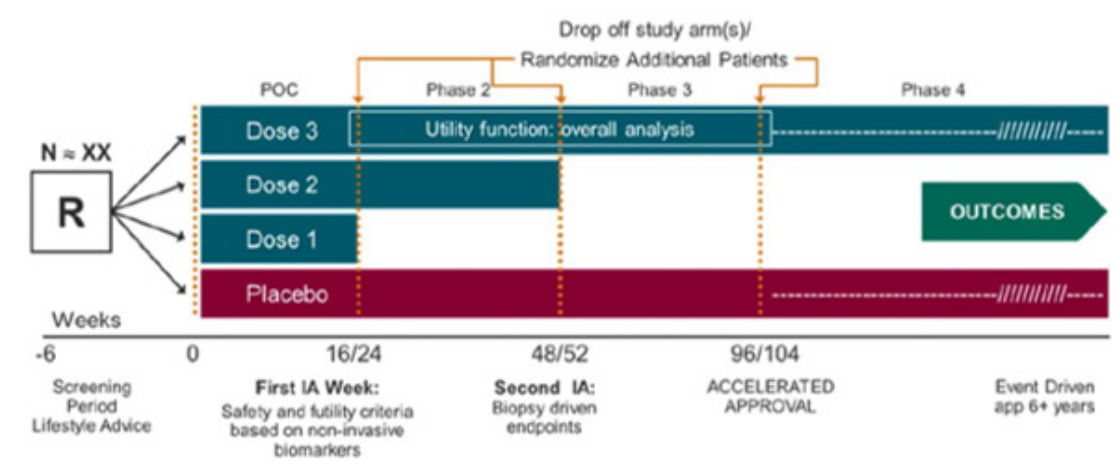

Figure 1: Phase 2/3/4 seamless adaptive design.

Because only one trial would lead to approval, a very small overall alpha (i.e., <0.001) is recommended to ensure proper control of a type I error.

Although the above seamless phase 2/3/4 appears to be reasonable, regulatory agency such as FDA emphasizes that the designs must be supported by a sound rationale and scientific justifiable for integrity, quality, and validity. Protocol should address the following typical issues:

I. Provide detailed information regarding how the overall type I error rate is controlled or preserved.

II. Provide a detailed strategy or plan for preventing possible operational biases that may incur before and after the adaptations are applied.

III. Provide justification regarding the validity of statistical methods used for a combined analysis.

IV. Provide justification for the chosen alpha spending function (e.g., O'Brien-Fleming) for stopping boundaries.

V. Provide justification regarding criteria used for critical decision-making at interims.

VI. Establish an independent data safety monitoring committee (IDMC) and provide IDMC charter.

VII. Provide justification for power analysis for sample size calculation and sample size allocation especially where the study objectives, endpoints, and populations are different at different stages.

VIII. Provide justification if sample size re-estimation is performed in a blinded or unblended fashion in the seamless adaptive trial design. 


\section{Conclusion}

There is unmet medical need for developing therapeutic strategies for patients with NASH. However, the long and bidirectional (i.e. some patients progressing but other ones regressing spontaneously) natural history of the disease and the requirement for sequential liver biopsies creates substantial challenges in clinical development. Up till now, despite considerable research and multiple clinical trials, at present there is no approved therapy. Thus, innovative study design which can not only help to speed up (or shorten) the development process but also to provide accurate and reliable assessment of the test treatment under investigation is needed.

The primary objective of the treatment for NASH is to prevent liver-related morbidity and mortality, which generally takes more than 10-20 years to develop. It is not feasible to perform such studies. Thus, the approach of considering surrogate endpoints for accelerated and conditional approval with a later confirmation of the positive impact of the compound in clinical outcomes is recommended. In this article, seamless adaptive clinical designs are recommended in NASH clinical trials. A seamless adaptive design cannot only provide an opportunity to shorten the overall path to registration for a new drug, but also offers continuity for patients enrolled in the study. Most importantly, the use of multiple-stage seamless adaptive trial designs (e.g., phase $2 / 3$, phase $3 / 4$, or phase $2 / 3 / 4$ seamless adaptive design) in NASH clinical trials is able to address the challenging issue that the study objectives, endpoints, and populations may be different from stage to stage and provide accurate and reliable assessment of the test treatment under investigation.

When the study endpoints are different at different stage, [11] proposed a therapeutic index function for analysis of two-stage seamless adaptive designs with distinct study endpoints at each stage under the assumption that even though the two endpoints are not the same, there is well-established relationship between them. A therapeutic index function is defined for each one of the endpoints (often consists of a number of criteria). It takes different endpoints with pre-specified criteria into consideration and it is based on a vector of therapeutic index function rather than individual endpoints. The vector of therapeutic index model allows the investigator to accurately and reliably assess the treatment effect in a more efficient way. Note that for NASH clinical trials utilizing complex innovative designs such as multiple-stage seamless adaptive designs with additional adaptions as described in [7] and [10], there are still many limitations and/or challenging issues that remain unsolved. For example, just to name a few, (i) valid statistical methods may not be fully developed, (ii) power calculation for sample size estimation and allocation at different stages may not be available, and (iii) the overall type I error rate may not be controlled. In these cases, further research is needed $[23,24]$.

\section{Acknowledgement}

None.

\section{Conflict of Interest}

No conflict of interest.

\section{References}

1. ZM, Koenig AB, Abdelatif D, Fazel Y, Henry L, et al. (2016) Global epidemiology of non-alcoholic fatty liver disease-meta-analytic assessment of prevalence, incidence, and outcomes. Hepatology 64: 7384.

2. Sanyal AJ (2011) NASH: A global health problem. Hepatol Res 41: 670674.

3. Sanyal A, Brunt E, Kleiner D, Kowdley KV, Chalasani N, et al. (2011) Endpoints and clinical trial design for nonalcoholic steatohepatitis. Hepatology 54: 344-353.

4. Kleiner DE, Brunt EM, Van Natta M, Behling C, Contos MJ, et al. (2005) Nonalcoholic Steatohepatitis Clinical Research Network. Design and validation of a histological scoring system for nonalcoholic fatty liver disease. Hepatology 41: 1313-1321.

5. Brunt EM, Kleiner DE, Wilson LA, Belt P, Neuschwander-Tetri BA (2011) NASH Clinical Research Network (CRN). Nonalcoholic fatty liver disease (NAFLD) activity score and the histopathologic diagnosis in NAFLD: distinct clinicopathologic meanings. Hepatology 53: 810-820.

6. FDA (2018) Guidance for Industry - Noncirrhotic Nonalcoholic Steatohepatitis With Liver Fibrosis: Developing Drugs for Treatment. The United States Food and Drug Administration, Silver Spring, Maryland.

7. Chow SC, Chang M (2011) Adaptive Design Methods in Clinical Trials. CRC Press: Chapman and Hall (eds.), ( $2^{\text {nd }}$ edn.), Taylor \& Francis, New York, USA.

8. Chow SC (2020) Innovative Methods for Rare Disease Drug Development. CRC Press: Chapman, Hall (eds.), Taylor \& Francis, New York, USA.

9. Chow SC, Corey R (2011) Benefits, Challenges, and obstacles of adaptive designs in clinical trials. The Orphanet Journal of Rare Diseases 6: 79.

10. FDA (2019) Guidance for Industry - Adaptive Designs for Clinical Trials of Drugs and Biologics. The United States Food and Drug Administration, Silver Spring, Maryland.

11. Filozof C, Chow SC, Dimick-Santos L, Chen YF, Williams RN, et al. (2017) Clinical endpoints and adaptive clinical trials in precirrhotic nonalcoholic steatohepatitis: facilitating development approaches for an emerging epidemic. Hepatology Communications 1: 577-585.

12. FDA (2014) Guidance for Industry - Expedited Programs for Serious Conditions - Drugs and Biologics. The United States Food and Drug Administration, Silver Spring, Maryland.

13. EMA (2014) Pilot project on adaptive licensing. European Medicines Agency, London, UK.

14. Sheiner L (1997) Learning versus confirming in clinical drug development. Clin Pharmacol Ther 61: 275-291.

15. Lominadze Z, Harrison S, Charlton M, Loomba R, Neuschwander-Tetri B, et al. (2014) Survey of diagnostic and treatment patterns of NAFLD and NASH in the United States: real life practices differ from published guidelines. Program and Abstracts of the 65th Annual Meeting of the American Association for the Study of Liver Diseases (AASLD).

16. Musso G, Gambino R, Cassader M, Pagano G (2011) Meta-analysis: natural history of non-alcoholic fatty liver disease (NAFLD) and diagnostic accuracy of non-invasive tests for liver disease severity. Annals of Medicine 43(8): 617-649.

17. Ekstedt M, Franzen LE, Mathiesen UL, Thorelius L, Holmqvist M, et al (2006) Long-term follow-up of patients with NAFLD and elevated liver enzymes. Hepatology 44: 865-873. 
18. Angulo P, Kleiner DE, Dam-Larsen S, Adams LA, Bjornsson ES, et al. (2015) Liver fibrosis, but no other histologic features, is associated with long-term outcomes of patients with nonalcoholic fatty liver disease. Gastroenterology 149(2): 389-397.e10.

19. Argo CK, Northup PG, Al-Osaimi AM, Caldwell SH (2009) Systematic review of risk factors for fibrosis progression in non-alcoholic steatohepatitis. J Hepatol 51: 371-379.

20. Singh S, Allen AM, Wang Z, Prokop LJ, Murad MH, et al. (2015) Fibrosis progression in nonalcoholic fatty liver vs nonalcoholic steatohepatitis: a systematic review and meta-analysis of paired biopsy studies. Clin Gastroenterol Hepatol 13: 643-54. e1-9.

21. Chang M (2007) Adaptive design method based on sum of p-values. Statistics in Medicine 26: 2772-2784.
22. Chow SC, Lu Q Tse SK (2007) Statistical analysis for two-stage adaptive design with different study endpoints. Journal of Biopharmaceutical Statistics 17: 1163-1176.

23. Filozof C, Goldstein BJ, Williams RN, Sanyal A (2015) Non-alcoholic steatohepatitis: limited available treatment options but promising drugs in development and recent progress towards a regulatory approval pathway. Drugs 75: 1373-1392.

24. Pagadala MR, McCullough AJ (2012) The relevance of liver histology to predicting clinically meaningful outcomes in nonalcoholic steatohepatitis. Clin Liv Dis 16: 487-504. 